\title{
Evaluation of Honed Cylinder Bores
}

\author{
F. Puente León \\ Design of Systems on Silicon (DS2), Parque Tecnológico de Valencia, \\ C./ Charles Robert Darwin 2, E-46980 Paterna (Valencia), Spain \\ Submitted by G. Spur (1), Berlin, Germany
}

\begin{abstract}
The quality of the honing texture on cylinder bores of combustion engines plays an important role with respect to oil consumption, noxious emissions, and running performance. To evaluate honed surfaces objectively, features describing the surface texture are extracted from 2-D data of the surface. The paper focuses on two crucial stages of the data analysis: the preprocessing, which aims at suppressing irrelevant components and enhancing the information of interest, and the feature extraction, which yields reliable numerical estimates of the surface characteristics of interest, like the honing angle, groove parameters, surface defects etc. The assessment results can easily be adapted to user-specific ratings.
\end{abstract}

Keywords: Honing, Surface texture, Automated visual inspection

\section{INTRODUCTION}

Cylinder bores of combustion engines are finished by honing. The resulting surface texture mainly consists of two bands of helical grooves placed stochastically and appearing at different angles to the cylinder axis. The texture quality is highly important for dry operation properties, oil consumption, noxious emissions, and running performance. Up to now, experts are still rating honed surfaces visually based on microscopic images. This method is tedious, subjective, and time consuming. To get objective and reproducible results, an automated method of inspection is necessary.

\section{INSPECTION APPROACH}

\subsection{Surface data}

There are basically different ways to measure the texture of a honed surface; see Table 1. Typically, a mechanical stylus only performs a 1-D measurement of the surface profile. In contrast to this, grey level images and optical profilometers provide 2-D data in a reasonable amount of time.

Because the lateral-geometric features of honing textures can only be analysed with 2-D data, in the following we will concentrate on such kinds of data. Other characteristics related to the different measurement principles investigated are also included in this table.

A signal model describing the essential characteristics of a honing texture constitutes the basis of the evaluation approach presented in this paper. Based on this model, clear and mathematically well-defined features are introduced, which enable a reproducible and objective assessment of the texture. This strategy differs from many popular methods-such as those relying on neural networks-, which are often treated as a 'black box' [1].

The features chosen are inspired by the Honing Atlas [2], by many opinions of experts, and have also been extended by adding new volumetric parameters for the case of analysing profile data. This results in an extensive set of features that can be customized to match the needs of individual users.

\subsection{Properties of honing textures}

Figure 1 shows some of the properties of honing textures, based upon which features are to be defined. The most popular ones are the roughness parameters, such as those based on the Bearing Ratio Curve (Abbott Curve) [3], and $R_{\mathrm{a}}, R_{\mathrm{z}}$ and $R_{\max }$ [4]. However, dealing with honed surfaces, it is important to define features that take the lateral geometry into account. This way, most relevant texture peculiarities can be described, such as the honing angle, material smearings, groove interrupts, stray grooves, holes, foreign bodies, and flakes, as shown in Figure 1. In addition, features describing the balance of grooves, presence of plateaus, shape of grooves, cracks, residual turning grooves, and chatter marks are also needed.

\subsection{Automated inspection}

Figure 2 shows an overview of the abilities and aims of automated inspection in quality control applied to the honing process. A 2-D or 3-D sensor provides data $g(x)$ of the honed surface, where $\boldsymbol{x}=(x, y)^{\top} \in \mathbf{R}^{2}$ denotes the lateral spatial coordinates. The grey coloured blocks of the diagram are part of the sensor data processing. The outputs of the system can be used simply as a statement about surface quality, to give alarms causing an interrupt of the machining process, or it can be fed back via a controller to regulate the honing process, because the honing texture contains information about both functionality and also machining process -independently of the fact whether a post-honing brushing is performed or not.

In the following sections, we will focus on two crucial stages of the automated inspection: the preprocessing of the sensor data and the feature extraction, and we will give some examples to these steps.

\section{PREPROCESSING}

The goal of the preprocessing is to suppress irrelevant components, namely the inhomogeneities $i(\boldsymbol{x})$ and the disturbances $b(\boldsymbol{x})$, while enhancing the information of interest, i.e. the texture $t(\boldsymbol{x})$. In the case of acquisition of image data, the inhomogeneities $i(\boldsymbol{x})$ could be due to 


\begin{tabular}{|l|c|c|c|}
\hline & Mechanical stylus & Grey level image & Optical profilometry \\
\hline \multicolumn{1}{|c|}{ Measurement region } & 1-D & 2-D & 2-D \\
\hline Depth information & Yes & No & Yes \\
\hline Lateral geometric information & No & $\begin{array}{c}\text { Possible with } \\
\text { reasonable effort }\end{array}$ & Very time-consuming \\
\hline Covering the entire surface & Very time-consuming & High & High \\
\hline Computational processing expense & Low & Yes & Yes \\
\hline Non-contact measurement & No & No & Yes \\
\hline Standardized parameters & Yes & \\
\hline
\end{tabular}

Table 1: Comparison between mechanical stylus devices, grey level images, and optical profilometers.

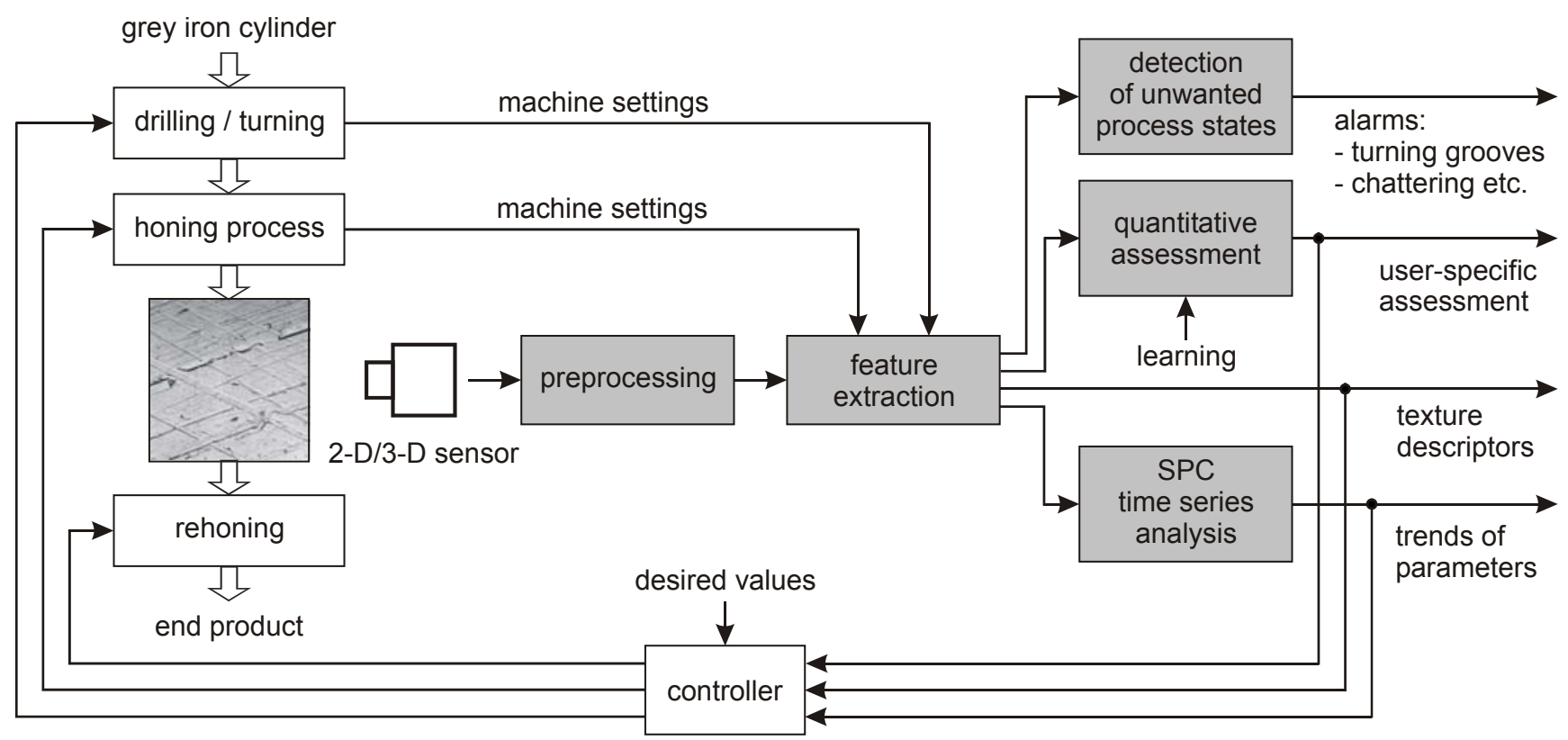

Figure 2: Automated inspection of honed surfaces.

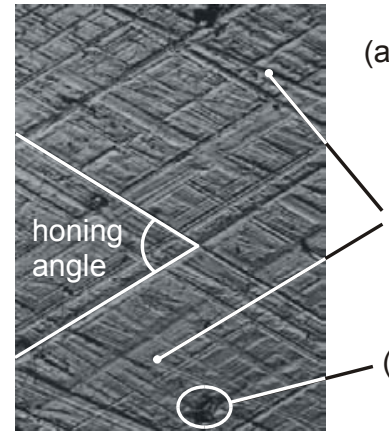

2-D grey level image (a)

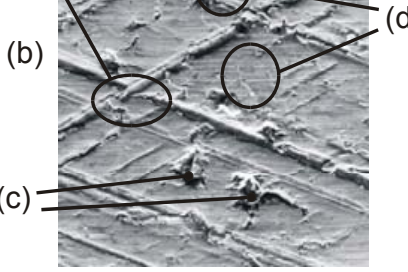

2-D SEM image

Figure 1: Honing textures showing lateral features and defects: (a) material smearings, groove interrupts;

(b) stray grooves; (c) holes or foreign bodies; (d) flakes.

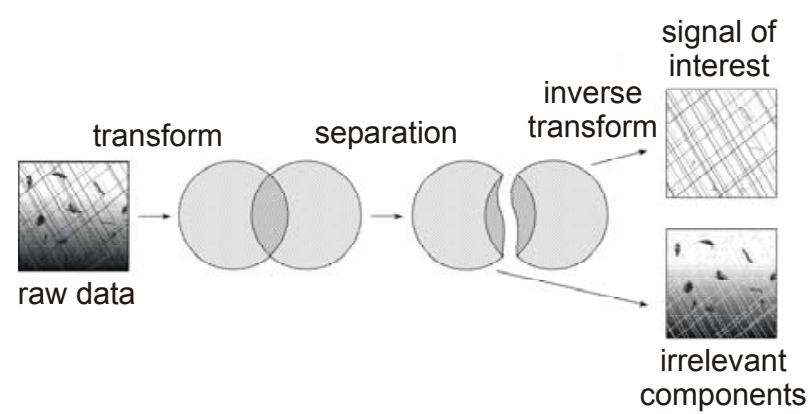

Figure 3: Principle of the preprocessing.

spatial variations of surface illumination. Other components assigned to the disturbances $b(\boldsymbol{x})$ include e.g. deviations from the ideal course of the grooves and defects, such as material smearings, flakes etc.

We use a signal model that describes the sensor data $g(\boldsymbol{x})$ as a combination of the texture $t(\boldsymbol{x})$ and the irrelevant components $i(\boldsymbol{x})$ and $b(\boldsymbol{x})$ :

$g(\mathbf{x})=f\{i(\mathbf{x}), b(\mathbf{x}), t(\mathbf{x})\}$.

To be able to recover the information of interest $t(\boldsymbol{x})$, an assumption is necessary: the different components have to be mathematically distinguishable.

As shown in Figure 3, a transform maps the raw data $g(\boldsymbol{x})$ such that a strict separation of their components is obtained. Then, the undesired components are suppressed, and finally an inverse transform is performed that yields the results of the preprocessing.

The benefits of this procedure include a simplification of the feature extraction, and a more robust image processing, as shown in the following examples.

\subsection{Homogenization}

When a groove texture is degraded by an intensity inhomogeneity $i(\boldsymbol{x})$ due to the data acquisition process, e.g. due to an inhomogeneous lighting, a homogenization can be performed to suppress this unwanted component [6]. Figure 4 shows an example of this operation for a planing texture. On the left side of the figure, the original texture is shown. The central image represents the result of a standard homogenization method-the homomorphic 

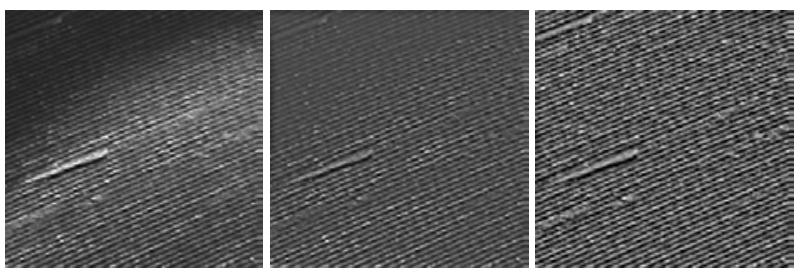

Figure 4: Homogenization: (left) planing texture; (centre) homomorphic filtering; (right) homogenization result.

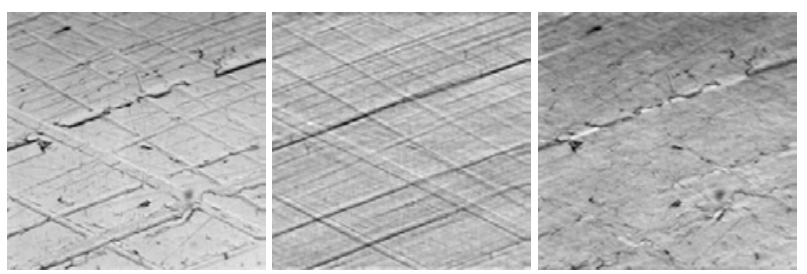

Figure 5: Texture decomposition: (left) honing texture; (centre) groove texture; (right) background texture.

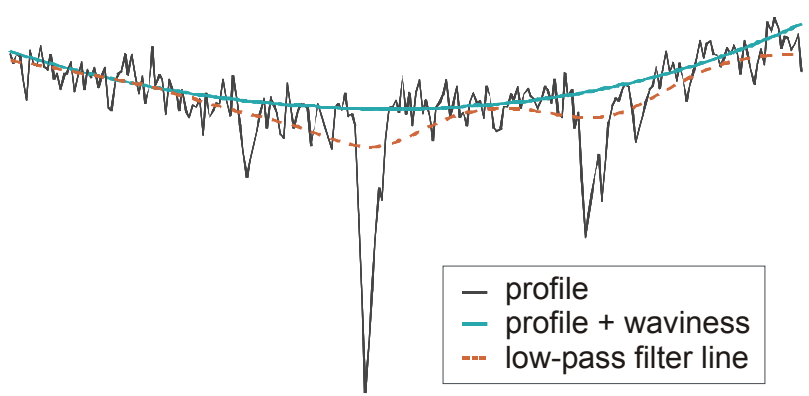

Figure 6: Reference surface: problems with conventional low-pass filters.

filtering, which assumes a multiplicative combination of texture and inhomogeneity. Especially in the upper left corner, this image shows a very poor contrast. The image on the right results from the model-based approach according to Figure 3. In this case, a homogenization of the local mean value and the local contrast has been performed based on a model that considers a mixed additive and multiplicative combination of both signal of interest and disturbing inhomogeneity [6]. The result is clearly more homogeneous than the former one and enables a more robust analysis of the texture.

\subsection{Texture decomposition}

The next example concerns the decomposition of the honing texture to ease the feature extraction. Due to the complexity of the honing texture, the extraction of relevant features needed for the inspection task could be simplified considerably, if the partial textures constituting the signal $g(\boldsymbol{x})$ according to Eq. (1) were available. Thus, it would be advantageous to develop a method to separate the texture $g(\boldsymbol{x})$ into a component $t(\boldsymbol{x})$ containing the straight structures (i.e. the grooves) and another one $b(\boldsymbol{x})$ showing the isotropic components (i.e. the background, including defects and objects). In this case, a homogeneous texture will be assumed.

Fortunately, a very efficient algorithm to perform this separation already exists [7]. The left side of Figure 5 shows an original honing texture; the other two images represent the results of the adaptive texture decomposition computed with this algorithm. In the groove texture, only the ideal grooves can be seen, whereas the background image contains all deviations from the ideal groove course as well as defects and other objects. For a more comprehensive discussion of the separation algorithm, interested readers are referred to [7].

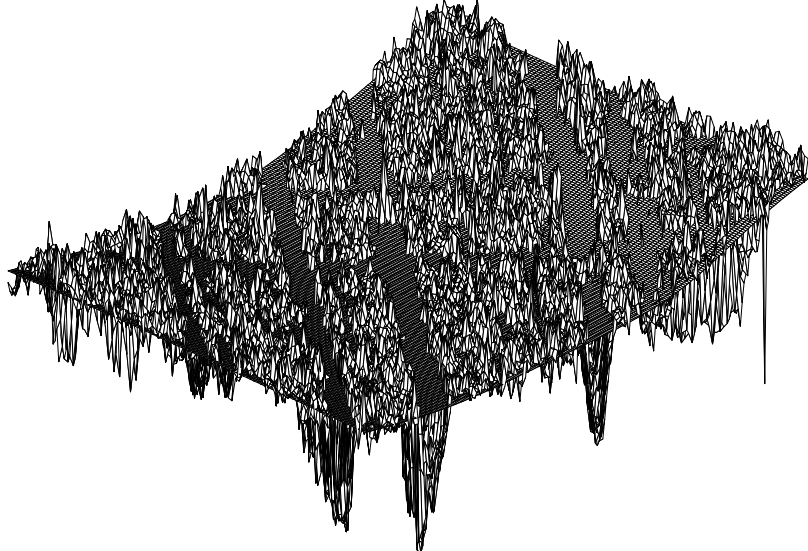

Figure 7: Original honed surface and reference surface.

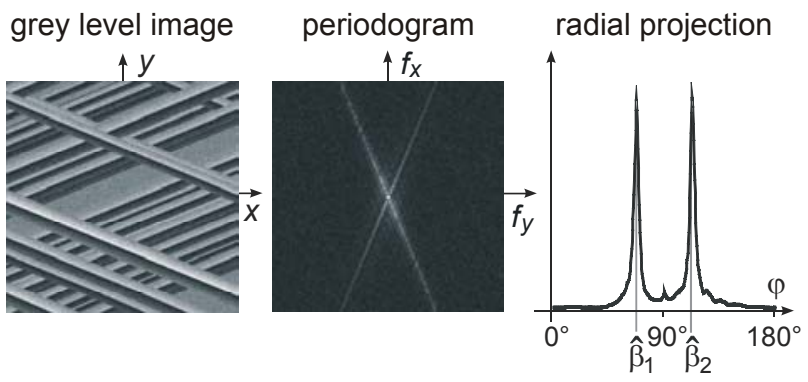

Figure 8: Estimation of the honing angle.

\subsection{Reference surface}

Finally, the definition of a reference surface to eliminate the shape component will be presented. The graph in Figure 6 represents a trace through the profile of a honed surface. The smooth line describes the shape component to be suppressed. However, conventional low-pass filters lead to distortions in the area of the grooves, as shown in the case of the dashed line. We have faced this problem by developing an iterative 2-D filter-a modified Gaussian filter-which behaves robustly even in case of deep grooves [8]. The 3-D plot depicted in Figure 7 shows a section of a honed surface as well as the resulting reference surface computed with this method.

\section{FEATURE EXTRACTION}

\subsection{Honing angle}

The first example of the feature extraction is the estimation of the honing angle. To this end, the periodogram (PG) is computed, which is proportional to the squared magnitude of the Fourier transform of the texture $g(x)$ :

$\hat{\Psi}_{g g}(\boldsymbol{f}) \propto|F\{g(\boldsymbol{x})\}|$.

The $P G$ is an estimator of the power spectral density (PSD) function, which specifies the spectral properties of the stochastic process generating the texture [9]. Then, the PG is projected radially; see Figure 8.

Since honing textures consist of two bands of grooves, the projection function also shows two pronounced maxima. The estimate of the honing angle results as the difference between the locations of both maxima:

$\hat{\beta}_{\mathrm{H}}=\left|\hat{\beta}_{2}-\hat{\beta}_{1}\right|$.

Despite the variance of the $P G$, due to the averaging performed, the radial projection is a very smooth curve. Thus, this procedure yields a fast and statistically reliable estimate for the honing angle. 


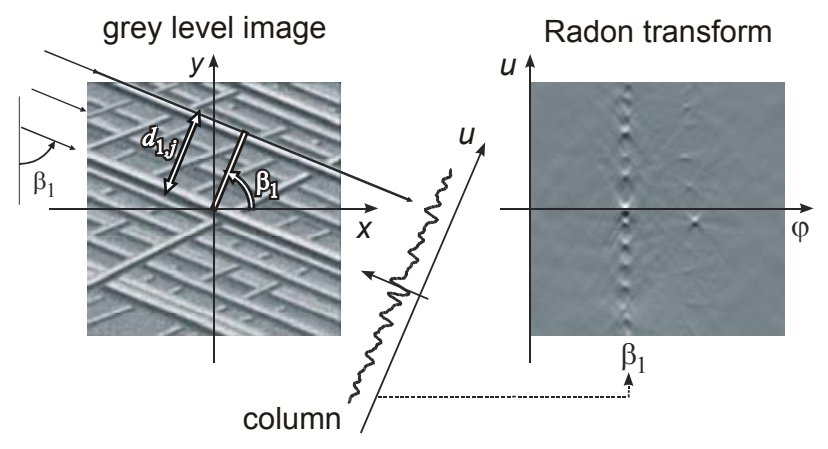

Figure 9: Illustration of the Radon transform.

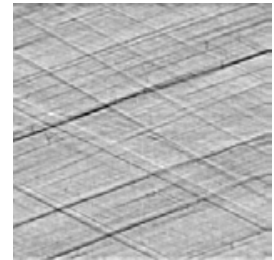

(a)

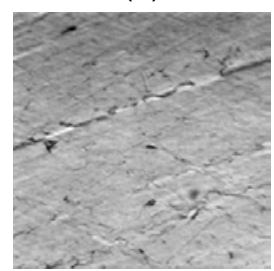

(d)

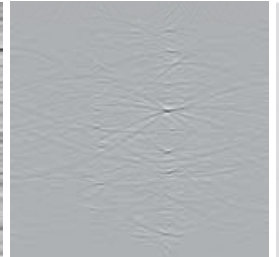

(b)

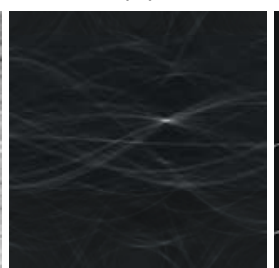

(e)

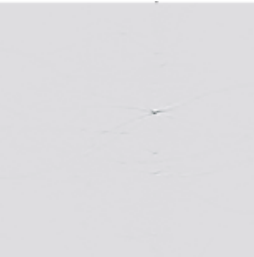

(c)

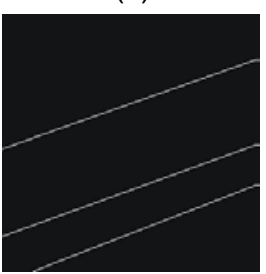

(f)
Figure 10: Detection of defects: (a) groove image; (b) Radon transform of (a); (c) multiplication of (b) and (e); (d) background image; (e) Radon transform of (d); (f) defective grooves detected.

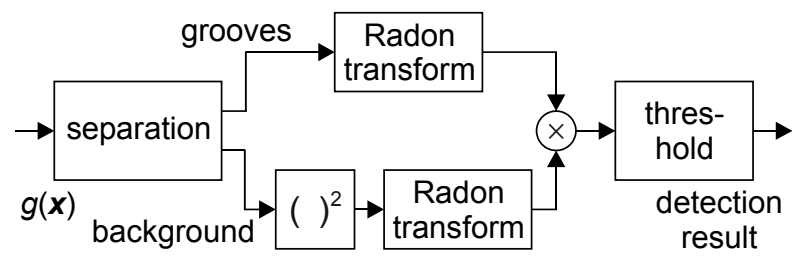

Figure 11: Algorithm to detect defective grooves.

\subsection{Groove parameters}

The next example concerns the extraction of the groove parameters. This is accomplished based on the Radon transform, which maps each line of a 2-D image onto a point of the transformation domain; as demonstrated in Figure 9 [10]. Following, all distinct peaks of the Radon transform, which correspond with grooves, are detected by means of morphological filters. Finally, for each detected groove, the corresponding parameters (amplitude, width, location, and angle) are estimated based on the output of the morphological filters [9].

\subsection{Detection of defects}

In Section 3.2, an algorithm enabling a decomposition of honing textures has been presented. This section focuses on the background texture obtained, which contains the main information concerning defects and objects, and discusses a robust approach allowing a detection of defects based on this image. It represents a refinement of the detection of grooves presented in the last subsection; see Figure 11 [11]. In this case, a Radon transform of the groove image Figure 10(a) obtained after decomposition is performed to concentrate the information concerning grooves; see Figure 10(b). Furthermore, collinear defects distributed along grooves are also concentrated by means of a Radon transform of the background image onto peaks in the Radon domain; see Figures $10(\mathrm{~d})$ and (e). By combining both groove texture and background texture in the Radon domain multiplicatively, only the peaks representing defective grooves remain; see Figure 10(c). The most pronounced peaks in this image correspond with the locations of the three grooves sketched in Figure 10(f), which are indeed the most salient defective grooves of the original image; see Figure 5(a).

\section{SUMMARY AND CONCLUSIONS}

This paper has shown how signal processing methods can be used to automatically evaluate relevant properties of the honing texture of grey iron cylinders with regard to different quality aspects. A preprocessing strategy has been presented that enables a robust automated assessment. Moreover, a feature-oriented approach has been proposed, in which the features are clear and mathematically well defined. By incorporating depth data, new function-relevant parameters can be computed.

In previous approaches, only roughness parameters from first-order statistics have been used to quantify the features of interest. The presented strategy, however, is based on an analysis of the essential lateral-geometric characteristics of the texture, including those related to higher-order statistics. This enables to automate and objectify the assessment proposed by experts and standards used in different companies.

[1] Malburg, M.C., Raja, J., 1993, Characterization of surface texture generated by plateau honing process, CIRP Annals, 42/1:637-640.

[2] AE Goetze GmbH, Burscheid, Germany, 1993, AE Goetze Honing Guide - Rating Criteria for the Honing of Cylinder Running Surfaces.

[3] DIN EN ISO 13565-2, 1996, Geometrical Product Specification (GPS) - Surface texture: Profile method; Surfaces having stratified functional properties - Part 2: Height characterization using the linear material ratio curve.

[4] DIN 4768, 1990, Determination of roughness parameters $R_{a}, R_{z}, R_{\max }$ by means of stylus instruments; terminology; measuring conditions.

[5] Pfeifer, T., Wiegers, L., 1998, Adaptive control for the optimized adjustment of imaging parameters for surface inspection using machine vision, CIRP Annals, 47/1:487-490.

[6] Beyerer, J., Puente León, F., 1997, Suppression of Inhomogeneities in Images of Textured Surfaces, Optical Engineering, 36/1:85-93.

[7] Beyerer, J., Puente León, F., 1998, Adaptive Separation of Random Lines and Background, Optical Engineering, 37/10:2733-2741.

[8] Krahe, D., 2000, Zerstörungsfreie Prüfung der Textur gehonter und geschliffener Gegenlaufflächen, VDI Verlag, Düsseldorf.

[9] Beyerer, J., Krahe, D., Puente León, F., 2001, Characterization of Cylinder Bores, In: Metrology and Properties of Engineering Surfaces, E. Mainsah, J.A. Greenwood, and D.G. Chetwynd (eds.), Kluwer Academic Publishers, Boston, MA.

[10] Deans, S.R., 1983, The Radon transform and some of its applications, John Wiley \& Sons, New York.

[11] Beyerer, J., Puente León, F., 1997, Detection of Defects in Groove Textures of Honed Surfaces, Int. J. of Machine Tools \& Manufacture, 37/3:371-389. 